\title{
3 Stress und Fertilität
}

\author{
Wolfgang Harth
}

Unter psychosomatischen Gesichtspunkten konnte bisher ein Zusammenhang zwischen Stress, Stresshormonen und tendenzieller Einschränkung der Fertilität in einigen Studien nachgewiesen werden. Prolaktin und Neopterin sind Marker von Stressrespondern. Subgruppen von Stressrespondern mit unerfülltem Kinderwunsch zeigen signifikant höhere Werte bei den Stressparametern Prolaktin, Kortisol, FSH und dem immunologischen Marker Neopterin sowie gleichzeitig eine Subfertilität mit Einschränkung der Motilität, dem Hypoosmotischen Schwelltest und der Penetrationsfähigkeit der Spermien auf.

Die neuroendokrinologischen und neuroimmunologischen Unterschiede sind in testpsychologischen Fragebögen bei Stressrespondern mit einer signifikant höheren Reaktionskontrolle assoziiert. Dies bedeutet, dass Nicht-Stressresponder möglicherweise einen Fertilitätsvorteil haben. Hier stellt sich auch die zentrale Frage nach der primären bzw. sekundären Genese: Führt ein erhöhtes Bedürfnis nach Reaktionskontrolle zu verstärktem Stress, oder aber erhöhter Stress zu höherem Bedürfnis nach Reaktionskontrolle und damit möglicherweise zu einem Nachteil für die Zeugungsfähigkeit? Die Frage kann abschließend nicht beantwortet werden. Die Indikation zur Therapie ist aber in beiden Fällen gegeben.

Andererseits zeigen die testpsychologischen Instrumente bei Männern mit Fertilitätsstörungen (Oligo-, Krypto- und Azoospermie) in der Stressverarbeitung signifikant höhere Positivstrategien mit Ersatzbefriedigung, Entspannung und Ablenkung auf. Bei Männern mit Fertilitätsstörungen liegt möglicherweise besseres positives Coping mit Positivstrategien vor, wobei die Erfüllung und Befriedigung aktiver in der Lebensgestaltung und der Partnerschaft ausgleichend außerhalb eines erfüllten Kinderwunsches gesucht wird.

Zwischen psychosozialem Stress und Subfertilität besteht ein messbarer jedoch kontrovers diskutierter Zusammenhang, welcher auch neue Therapieoptionen beinhalten könnte. 
Bei Stressrespondern sollte zusätzlich zur medikamentösen Therapie ergänzend die Einleitung von Entspannungsmaßnahmen als weiterführende Kombinationstherapie zur Stresspufferung erfolgen.

\subsection{Sterile Ehen}

Sexualität in sterilen Ehen ist problematisch, jedoch lassen sich in zahlreichen Studien unter Einsatz von testpsychologischen Fragebögen teilweise nur widersprüchliche Ergebnisse aufzeigen.

\section{Partner in sterilen Ehen sind eine heterogene Gruppe ohne pauschal für alle Patienten charakteristische spezifische Persönlichkeitsauffälligkeiten.}

Im Durchschnitt aller Studien zeigen sich bei 78\% der Paare mit unerfülltem Kinderwunsch keine messbaren psychischen Auffälligkeiten.

Es scheint insgesamt ein heterogener Problembereich mit konflikthaften Untergruppen von Paaren vorzuliegen, bei denen psychische Auffälligkeiten mit Behandlungsbedarf bestehen. Hinzu kommt die Schwierigkeit primäre psychische Störungen und sekundär bedingte Folgestörungen zu unterscheiden.

Bei Paaren in sterilen Ehen scheint besonders das Selbstwertgefühl und die Sexualität beeinflusst zu sein (Bernstein et al. 1988). Wenn der Kinderwunsch nicht spontan verwirklicht wird, treten ernste Zweifel an der eigenen Vollkommenheit auf, zuerst bei der Frau, weil traditionelles Denken selbstverständlich eine Fertilität des Mannes vermuten lässt, solange Kohabitation und Ejakulation funktionieren.

Erfahren Männer von ihrer Infertilität im Vollbesitz ihrer sexuellen Potenz können schwere Störungen mit psychischem Schock und depressive Anpassungsstörungen auftreten. Es folgen Selbstbeschuldigungen, Schuldzuweisungen, Schuldempfinden vor der Partnerin, bis hin zu Instabilität der Paarbeziehung und Ehekrisen sowie Trennung. Libidomangel und Liebesentzug sind oftmals Folgen des frustrierenden Kinderwunsches.

Weitere Einzelbefunde zeigen eine höhere Depressivität, Ängstlichkeit und Somatisierungstendenz bei männlicher Infertilität (Conrad et al. 2003) aber auch ein höheres Ausmass an Alexithymie auf.

Es gibt dabei Hinweise auf zunehmende partnerschaftliche Unzufriedenheit bei zeitlich länger bestehender ungewollter Kinderlosigkeit (Brähler et al. 200o).

\subsection{Psychogene Sterilität}

Die rein psychogene Sterilität in der Ehe ist äußerst selten, wird aber immer wieder in der andrologischen Sprechstunde beobachtet, und stellt dann meist einen Überraschungsbefund dar. 
Eine eindeutige psychogene Sterilität liegt vor, wenn das Paar mit unerfülltem Kinderwunsch trotz ärztlicher Aufklärung

1. selbstschädigendes Verhalten (Abusus, Essstörung uä.) fortführt,

2. an fruchtbaren Tagen oder gar keine Kohabitation durchführt,

3. notwendige Maßnahmen zur Fertilitätsbehandlung zustimmt aber nicht durchführt.

So zeigt sich in Einzelfällen meist erst nach aufwendigen diagnostischen Maßnahmen, dass überhaupt nur selten und dann außerhalb der fruchtbaren Tagen eine Kohabitation erfolgte, ohne dass dies von dem Paar anfänglich in der Sprechstunde thematisiert werden konnte.

Als erste Therapiemaßnahme sollte eine Psychoedukation unter sexualtherapeutischen Gesichtspunkten erfolgen, sowie ggf. später auch eine Paartherapie in Betracht gezogen werden.

\subsection{Psychotherapie}

Allgemeine Hinweise zur Therapie sind aufgrund der Datenlage kaum zu treffen. Entspannungsverfahren, stützende psychosomatische Grundversorgung unter sexualmedizinischen Gesichtspunkten und gegebenenfalls eine weiterführende Einzel- oder Paartherapie meist als Fokaltherapie sind sinnvoll. Psychoanalytische Verfahren wurden in Studien bisher nicht untersucht, Verhaltenstherapie und Entspannungsverfahren scheinen wirksam zu sein. Für die individuelle Behandlung sind komplexe Strategien zum Teil in überregionalen Spezialsprechstunden notwendig. Dies beinhaltet auch eine Psychoedukation. Ziele dieser Sprechstunden sind eine Bewältigung auch Akzeptanz der Kinderlosigkeit, bessere Paarkommunikation und Hilfe bei der Suche nach Alternativen (Wischmann 2003).

\section{Literatur}

Bernstein I, Mattox JH, Keller R (1988) Psychological status of previously infertile couples after a successful pregnancy. Journal of Obstetrics, Gynecology and Neonatal Nursing 17, 404-408

Brähler E, Felder H, Strauß B (Hrsg.) (2000) Psychosoziale Aspekte der ungewollten Kinderlosigkeit. Jahrbuch der Medizinischen Psychologie Bd. 17. Göttingen: Hogrefe

Buddeberg C (1987) Sexualberatung, 2. überarbeitete und erweiterte Auflage, Ferdinand Enke Verlag, Stuttgart

Conrad R, Schilling G, Hagemann T, Haidl G, Liedtke R (2003) Somatization and alexithymia in male infertility. A replication study. Hautarzt 54, 530-535

Gollenberg AL, Liu F, Brazil C, Drobnis EZ, Guzick D, Overstreet JW, Redmon JB, Sparks A, Wang C, Swan SH (2010) Semen quality in fertile men in relation to psychosocial stress. Fertil Steril 93, 1104-1111

Harth W, Linse R (2004) Male Fertility: Endocrine stress-parameters and coping. Dermatol Psychosom 5, 22-29

Morelli G, De Gennaro L, Ferrara M, Dondero F, Lenzi A, Lombardo F, Gandini L (2000) Psychosocial factors and male seminal parameters. Biol Psychol 53, 1-11

Seikowski K, Starke K (2002) Sexualität des Mannes. Lengerich, Pabst Science Publishers

Strauß B, Bettge S, Bindt C, Brähler E, Felder H, Gagel D, Goldschmidt S, Henning K, Ittner E, Kentenich H, Ningel K, Stammer H, Verres R, Wischmann T, Yüksel E (2000) Leitlinien - Psychosomatik in der Reproduktionsmedizin. Reproduktionsmedizin (5), S. 326-331

Wischmann T (2003) Psychosoziale Aspekte bei Fertilitätsstörung. Gynäkologe 36, 125-136 